\title{
Passive-Damping Design for Vibration Control of Large Structures*
}

\author{
Francisco Palacios-Quiñonero ${ }^{1}$, Hamid R. Karimi ${ }^{2}$, Josep Rubió-Massegú ${ }^{1}$ and Josep M. Rossell ${ }^{1}$
}

\begin{abstract}
In this work, a systematic strategy to design passive damping systems for structural vibration control is presented. The proposed design methodology is based on the equivalence between decentralized static velocity-feedback controllers and passive damping systems. By using recent developments in static output-feedback control, the design of passivedamping systems can be formulated as a single optimization problem with Linear Matrix Inequality constraints. Moreover, this optimization problem can be efficiently solved with standard numerical tools, even for large dimension systems. Due to its computational effectiveness, the proposed methodology can be applied to the design of passive damping systems for large structures. To illustrate the main ideas and methods, a passive damping system is designed for the seismic protection of a five-story building with excellent results.
\end{abstract}

\section{INTRODUCTION}

Passive energy dissipation devices, such as viscous fluid dampers, viscoelastic dampers, friction dampers, etc., are simple, compact, and reliable. Effective and relatively inexpensive energy dissipation systems (EDSs) for vibration control of large structures can be designed by implementing a set of passive dampers at suitable locations of the structure [1]-[3]. Determining the damping capacity of the different dampers is a challenging problem associated to the design of passive EDSs. In traditional approaches to this problem, the dampers are assumed to be identical and trial-and-error procedures are used to determine the damping capacities.

When the damping force can be considered proportional to the velocity (as it happens for viscous fluid dampers, for example), the action of a fully decentralized static velocityfeedback control system is equivalent to the actuation of a passive damping system. This fact makes possible to take advantage of the powerful design tools of feedback control to compute the damping capacities [4]-[6]. The main difficulties associated to this new approach are mainly related to the computational cost of designing decentralized static output-feedback controllers, which has traditionally been based on iterative procedures.

Recently, an effective strategy to compute structured output-feedback control gain matrices by solving a single

\footnotetext{
*This work was partially supported by the Spanish Ministry of Economy and Competitiveness through the grant DPI2012-32375, and by the Norwegian Center of Offshore Wind Energy (NORCOWE) under grant 193821/S60 from the Research Council of Norway (RCN). NORCOWE is a consortium with partners from industry and science, hosted by Christian Michelsen Research.

${ }^{1}$ CoDAlab. Department of Applied Mathematics III, Universitat Politècnica de Catalunya (UPC). Av. Bases de Manresa 61-73, (08242) Manresa, Barcelona, Spain. E-mails: francisco.palacios lupc.edu, josep.rubio@upc.edu, josep.maria.rossell@upc.edu

${ }^{2}$ Department of Engineering, Faculty of Engineering and Science University of Agder (UiA), N-4898 Grimstad, Norway. E-mail: hamid.r.karimi@uia.no
}

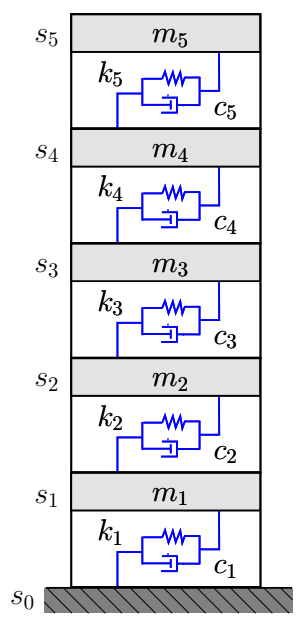

Fig. 1. Five-story building mechanical model

optimization problem with Linear Matrix Inequality (LMI) constraints was presented in [7]. The objective of the present paper is to apply these recent advances in output-feedback control to the design of passive EDSs for structural vibration control of large structures. For clarity and brevity, the main ideas are presented through the design of a passive EDS for the seismic protection of a five-story building.

The rest of the paper is organized as follows. Section II is devoted to discuss the equivalence between passive linear damping systems and fully decentralized static velocityfeedback controllers. In Section III, the new methodology to compute decentralized static output-feedback $H_{\infty}$ controllers is summarized. In Section IV, a passive damping system and a state-feedback $H_{\infty}$ controller are designed for the seismic protection of a five-story building. The statefeedback controller is taken as a reference in the performance assessment. Numerical simulations of the free and controlled vibrational responses of the five-story building together with the corresponding control actions are presented and compared in Section V. The full-scale North-South 1940 El Centro seismic record is used as ground acceleration in these numerical simulations. Finally, in Section VI, some future research directions are briefly discussed.

\section{PASSIVE DAMPING SYSTEMS AND DECENTRALIZED STATIC VELOCITY-FEEDBACK CONTROLLERS}

Let us consider the five-story building schematically depicted in Figure 1. The building motion can be described by the second-order differential equation

$$
M \ddot{q}(t)+C \dot{q}(t)+K q(t)=T_{u} u(t)+T_{w} w(t),
$$


where

$$
q(t)=\left[q_{1}(t), q_{2}(t), q_{3}(t), q_{4}(t), q_{5}(t)\right]^{T},
$$

is the vector of displacements relative to the ground, with $q_{i}(t), 1 \leq i \leq 5$, denoting the lateral displacement of the $i$ th story $s_{i}$ with respect to the ground level $s_{0} . M, C$, and $K$ are, respectively, the mass, damping, and stiffness matrices, which have the following structure:

$$
\begin{gathered}
M=\left[\begin{array}{ccccc}
m_{1} & 0 & 0 & 0 & 0 \\
0 & m_{2} & 0 & 0 & 0 \\
0 & 0 & m_{3} & 0 & 0 \\
0 & 0 & 0 & m_{4} & 0 \\
0 & 0 & 0 & 0 & m_{5}
\end{array}\right], \\
C=\left[\begin{array}{ccccc}
c_{1}+c_{2} & -c_{2} & 0 & 0 & 0 \\
-c_{2} & c_{2}+c_{3} & -c_{3} & 0 & 0 \\
0 & -c_{3} & c_{3}+c_{4} & -c_{4} & 0 \\
0 & 0 & -c_{4} & c_{4}+c_{5} & -c_{5} \\
0 & 0 & 0 & -c_{5} & c_{5}
\end{array}\right], \\
K=\left[\begin{array}{ccccc}
k_{1}+k_{2} & -k_{2} & 0 & 0 & 0 \\
-k_{2} & k_{2}+k_{3} & -k_{3} & 0 & 0 \\
0 & -k_{3} & k_{3}+k_{4} & -k_{4} & 0 \\
0 & 0 & -k_{4} & k_{4}+k_{5} & -k_{5} \\
0 & 0 & 0 & -k_{5} & k_{5}
\end{array}\right] .
\end{gathered}
$$

Between the consecutive stories $s_{i-1}$ and $s_{i}$, we assume that an actuation device $a_{i}$ has been implemented, which exerts a control action $u_{i}(t)$ as indicated in Figure 2. The vector of control actions is

$$
u(t)=\left[u_{1}(t), u_{2}(t), u_{3}(t), u_{4}(t), u_{5}(t)\right]^{T} .
$$

The control location matrix is

$$
T_{u}=\left[\begin{array}{rrrrr}
1 & -1 & 0 & 0 & 0 \\
0 & 1 & -1 & 0 & 0 \\
0 & 0 & 1 & -1 & 0 \\
0 & 0 & 0 & 1 & -1 \\
0 & 0 & 0 & 0 & 1
\end{array}\right]
$$

$w(t) \in \mathbb{R}$ denotes the seismic ground acceleration, and

$$
T_{w}=-M\left[\begin{array}{l}
1 \\
1 \\
1 \\
1 \\
1
\end{array}\right]
$$

is the excitation location matrix. Now, we consider the vector of interstory velocities

$$
v_{r}(t)=\left[\dot{q}_{1}, \dot{q}_{2}-\dot{q}_{1}, \dot{q}_{3}-\dot{q}_{2}, \dot{q}_{4}-\dot{q}_{3}, \dot{q}_{5}-\dot{q}_{4}\right]^{T},
$$

and assume that we are able to compute a fully decentralized static velocity-feedback controller

$$
u(t)=G_{d} v_{r}(t)
$$

with diagonal control gain matrix

$$
G_{d}=\left[\begin{array}{ccccc}
g_{11} & 0 & 0 & 0 & 0 \\
0 & g_{22} & 0 & 0 & 0 \\
0 & 0 & g_{33} & 0 & 0 \\
0 & 0 & 0 & g_{44} & 0 \\
0 & 0 & 0 & 0 & g_{55}
\end{array}\right]
$$

If the elements $g_{i i}$ are all negative, we can write

$$
\hat{c}_{i}=-g_{i i}, \quad 1 \leq i \leq 5,
$$

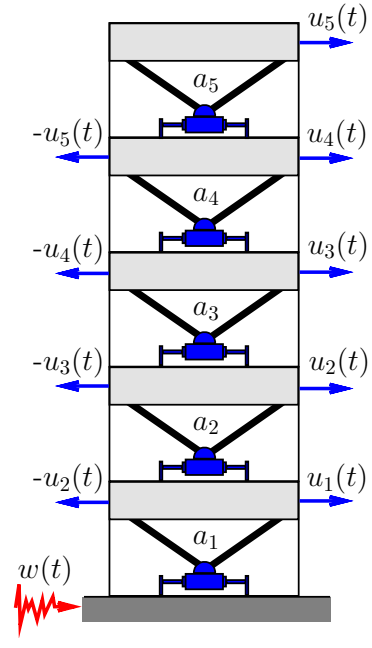

Fig. 2. Actuation system for the five-story building

and the vector of control forces

$$
f_{u}(t)=T_{u} u(t)
$$

takes the following form:

$$
f_{u}(t)=-\hat{C} \dot{q}(t)
$$

where

$$
\hat{C}=\left[\begin{array}{ccccc}
\hat{c}_{1}+\hat{c}_{2} & -\hat{c}_{2} & 0 & 0 & 0 \\
-\hat{c}_{2} & \hat{c}_{2}+\hat{c}_{3} & -\hat{c}_{3} & 0 & 0 \\
0 & -\hat{c}_{3} & \hat{c}_{3}+\hat{c}_{4} & -\hat{c}_{4} & 0 \\
0 & 0 & -\hat{c}_{4} & \hat{c}_{4}+\hat{c}_{5} & -\hat{c}_{5} \\
0 & 0 & 0 & -\hat{c}_{5} & \hat{c}_{5}
\end{array}\right]
$$

In this case, the system motion can be described by the second-order model

$$
M \ddot{q}(t)+(C+\hat{C}) \dot{q}(t)+K q(t)=T_{w} w(t),
$$

and the control forces can be implemented by taking the actuation devices $a_{i}$ as linear passive dampers with damping constants $\hat{c}_{i}$.

\section{Design OF STATIC OUTPUT-FEEDBACK $H_{\infty}$ CONTROLLERS}

Obviously, to apply the approach discussed in the previous section, an effective methodology to compute decentralized velocity-feedback controllers is required. In this context, the new design strategy for static output-feedback control proposed in [7] can be an excellent tool. In this section we summarize the main elements of this design strategy for $H_{\infty}$ controllers; a more detailed discussion can be found in [7] and $[8]$.

Let us consider the system

$$
\mathcal{S}:\left\{\begin{array}{l}
\dot{x}(t)=A x(t)+B u(t)+E w(t), \\
y(t)=C_{y} x(t) \\
z(t)=C_{z} x(t)+D_{z} u(t)
\end{array}\right.
$$

where $x(t) \in \mathbb{R}^{n}$ is the state, $u(t) \in \mathbb{R}^{m}$ is the control input, $w(t) \in \mathbb{R}^{r}$ is the disturbance input, $y(t) \in \mathbb{R}^{p}$ is the observed output, and $z(t) \in \mathbb{R}^{n_{z}}$ is the controlled output. $A, B, E$, 


$$
\begin{gathered}
{\left[\begin{array}{cr}
A X+X A^{T}+B G C_{y} X+X C_{y}^{T} G^{T} B^{T}+\gamma^{-2} E E^{T} & * \\
C_{z} X+D_{z} G C_{y} X & -I
\end{array}\right]<0 \quad \text { (MI-A) }} \\
{\left[\begin{array}{cr}
A Q X_{Q} Q^{T}+Q X_{Q} Q^{T} A^{T}+A R X_{R} R^{T}+R X_{R} R^{T} A^{T}+B Y_{R} R^{T}+R Y_{R}^{T} B^{T}+\eta E E^{T} & * \\
C_{z} Q X_{Q} Q^{T}+C_{z} R X_{R} R^{T}+D_{z} Y_{R} R^{T} & -I
\end{array}\right]<0 \quad \text { (LMI-B) }}
\end{gathered}
$$

Fig. 3. Matrix inequalities

$C_{y}, C_{z}$, and $D_{z}$ are known, real and constant matrices of appropriate dimensions. A static output-feedback controller has the form

$$
u(t)=G y(t),
$$

where $G$ is a constant control gain matrix. From (17) and (18), we obtain the following closed-loop system:

$$
\mathcal{S}_{C L}:\left\{\begin{array}{l}
\dot{x}(t)=\bar{A}_{G} x(t)+E w(t), \\
z(t)=\bar{C}_{G} x(t)
\end{array}\right.
$$

where

$$
\bar{A}_{G}=A+B G C_{y}, \quad \bar{C}_{G}=C_{z}+D_{z} G C_{y} .
$$

The $H_{\infty}$ control approach considers the largest energy gain from disturbance to controlled output

$$
\gamma_{G}=\sup _{\|w\|_{2} \neq 0} \frac{\|z\|_{2}}{\|w\|_{2}}
$$

where $w(t)$, and $z(t)$ denote, respectively, the disturbance input and controlled output in (17), and $\|\cdot\|_{2}$ is the usual continuous 2-norm

$$
\|f\|_{2}=\left[\int_{0}^{\infty}\{f(t)\}^{T} f(t) d t\right]^{1 / 2} .
$$

The control design objective is to obtain a gain matrix $\tilde{G}$ which simultaneously produces a stable closed-loop matrix $\bar{A}_{\tilde{G}}$ and an optimally small value $\gamma_{\tilde{G}}$. Using the closed-loop transfer function from the disturbance $w(t)$ to the controlled output $z(t)$

$$
T_{G}(s)=\bar{C}_{G}\left(s I-\bar{A}_{G}\right)^{-1} E,
$$

the value $\gamma_{\tilde{G}}$ can be expressed as the $H_{\infty}$-norm of $T_{\tilde{G}}$

$$
\gamma_{\tilde{G}}=\left\|T_{\tilde{G}}(s)\right\|_{\infty}=\sup _{\omega} \bar{\sigma}\left[T_{\tilde{G}}(j \omega)\right],
$$

where $\bar{\sigma}[\cdot]$ denotes the maximum singular value.

According to the Bounded Real Lemma [9], for a prescribed $\gamma>0$, the following two statements are equivalent:

1) $\left\|T_{G}(s)\right\|_{\infty}<\gamma$, and $\bar{A}_{G}$ is stable.

2) There exists a symmetric positive-definite matrix $X \in \mathbb{R}^{n \times n}$ such that the matrix inequality

$$
\left[\begin{array}{cr}
\bar{A}_{G} X+X \bar{A}_{G}^{T}+\gamma^{-2} E E^{T} & * \\
\bar{C}_{G} X & -I
\end{array}\right]<0
$$

holds, where $*$ denotes the transpose elements in the symmetric positions.
From (20) and (25), we obtain the nonlinear matrix inequality (MI-A) displayed in Figure 3, which can be converted into the following LMI:

$$
\left[\begin{array}{cr}
A X+X A^{T}+B Y+Y^{T} B^{T}+\eta E E^{T} & * \\
C_{z} X+D_{z} Y & -I
\end{array}\right]<0
$$

by introducing the new variables

$$
Y=G C_{y} X, \quad \eta=\gamma^{-2} .
$$

The continuous-time output-feedback $H_{\infty}$ control design problem can now be formulated as the following optimization problem:

$$
\left\{\begin{array}{l}
\text { maximize } \eta \\
\text { subject to } X>0, \eta>0 \text { and the LMI in (26), }
\end{array}\right.
$$

where the matrices $X$ and $Y$ are the optimization variables. If an optimal value $\eta_{\text {opt }}$ is attained for the matrices $\tilde{X}, \tilde{Y}$, and a control matrix $\tilde{G}$ satisfying

$$
\tilde{Y}=\tilde{G} C_{y} \tilde{X}
$$

can be determined, then the corresponding static outputfeedback controller

$$
u(t)=\tilde{G} y(t)
$$

defines a stable closed-loop matrix $\bar{A}_{\tilde{G}}$ with an associated $H_{\infty}$-norm

$$
\gamma_{\tilde{G}}=\left(\eta_{\mathrm{opt}}\right)^{-1 / 2} .
$$

It should be noted that equation (29) provides an implicit definition of the gain matrix $\tilde{G}$ and, in general, this equation can not be properly solved to obtain $\tilde{G}$. Using the transformations of the LMI variables proposed in [7], a simple and explicit formulation for the gain matrix $\tilde{G}$ can be obtained. Moreover, decentralized static output-feedback controllers can also be designed by imposing an appropriate zero-nonzero structure on the new LMI variables. Next, we summarize the main ideas of this design strategy.

Given a full row-rank output matrix $C_{y}$ with dimensions $p \times n, p \leq n$, we consider an $n \times(n-p)$ matrix $Q$, whose columns are a basis of $\operatorname{Ker}\left(C_{y}\right)$; and the Moore-Penrose pseudo-inverse of $C_{y}$, which is given by

$$
R=C_{y}^{T}\left(C_{y} C_{y}^{T}\right)^{-1} .
$$

From matrices $Q$ and $R$, we define the following transformations:

$$
X=Q X_{Q} Q^{T}+R X_{R} R^{T}, \quad Y=Y_{R} R^{T},
$$


where $X_{Q}, X_{R}$ are symmetric positive-definite matrices with respective dimensions $(n-p) \times(n-p), p \times p$; and $Y_{R}$ is an $m \times p$ matrix. Using the transformations (33), the LMI (26) takes the form (LMI-B) displayed in Figure 3. If the following optimization problem

$$
\left\{\begin{array}{l}
\text { maximize } \eta \\
\text { subject to } X_{Q}>0, X_{R}>0, \eta>0, \text { and (LMI-B), }
\end{array}\right.
$$

is solvable with an optimum value $\tilde{\eta}_{\text {opt }}$ attained by the matrices $\tilde{X}_{Q}, \tilde{X}_{R}$, and $\tilde{Y}_{R}$, then the control matrix

$$
\tilde{G}=\tilde{Y}_{R}\left(\tilde{X}_{R}\right)^{-1}
$$

defines a static output-feedback controller

$$
u(t)=\tilde{G} y(t)
$$

with stable closed-loop matrix $\bar{A}_{\tilde{G}}$, and $H_{\infty}$-norm

$$
\gamma_{\tilde{G}} \leq\left(\tilde{\eta}_{\mathrm{opt}}\right)^{-1 / 2}
$$

\section{DESIGN OF THE PASSIVE DAMPING SYSTEM}

In this section, the ideas presented in Section II and Section III are applied to the design of a passive damping system for the seismic protection of a five-story building. In Subsection IV-A, a first-order state-space model of the building is provided. In Subsection IV-B, a static statefeedback $H_{\infty}$ controller is designed, which will be taken as a reference in the performance assessment of the proposed passive damping system. Finally, in Subsection IV$\mathrm{C}$, a decentralized static velocity-feedback $H_{\infty}$ controller is designed to compute the damping capacities for the passive damping system.

\section{A. State-space building model}

Let us consider the second-order model (1) with the matrices $T_{u}$ and $T_{w}$ given in (7) and (8), respectively, and the following mass, damping, and stiffness matrices:

$$
\begin{gathered}
M=10^{3} \times\left[\begin{array}{ccccc}
215.2 & 0 & 0 & 0 & 0 \\
0 & 209.2 & 0 & 0 & 0 \\
0 & 0 & 207.0 & 0 & 0 \\
0 & 0 & 0 & 204.8 & 0 \\
0 & 0 & 0 & 0 & 266.1
\end{array}\right], \quad(38) \\
C=10^{3} \times\left[\begin{array}{cccccc}
260.2 & -92.4 & 0 & 0 & 0 \\
-92.4 & 219.6 & -81.0 & 0 & 0 \\
0 & -81.0 & 199.5 & -72.8 & 0 \\
0 & 0 & -72.8 & 186.7 & -68.7 \\
0 & 0 & 0 & -68.7 & 127.4
\end{array}\right], \\
K=10^{6} \times\left[\begin{array}{rrrrr}
260 & -113 & 0 & 0 & 0 \\
-113 & 212 & -99 & 0 & 0 \\
0 & -99 & 188 & -89 & 0 \\
0 & 0 & -89 & 173 & -84 \\
0 & 0 & 0 & -84 & 84
\end{array}\right], \quad(40)
\end{gathered}
$$

where masses are in $\mathrm{kg}$, damping coefficients in $\mathrm{Ns} / \mathrm{m}$, and stiffness coefficients in $\mathrm{N} / \mathrm{m}$. The mass and stiffness values in (38) and (40) are similar to those corresponding to the Kajima-Sizuoka building presented in [10]; the damping matrix $C$ has been computed as a Rayleigh damping matrix with a $2 \%$ damping ratio on the first and fifth modes [11]. By taking the state vector

$$
x_{I}(t)=\left[\begin{array}{c}
q(t) \\
\dot{q}(t)
\end{array}\right]
$$

we can derive a first-order state-space model

$$
S_{I}: \dot{x}_{I}(t)=A_{I} x_{I}(t)+B_{I} u(t)+E_{I} w(t),
$$

with state matrix

$$
A_{I}=\left[\begin{array}{cc}
{[0]_{5 \times 5}} & I_{5} \\
-M^{-1} K & -M^{-1} C
\end{array}\right]
$$

and the following control input and disturbance input matrices:

$$
B_{I}=\left[\begin{array}{c}
{[0]_{5 \times 5}} \\
M^{-1} T_{u}
\end{array}\right], \quad E_{I}=\left[\begin{array}{c}
{[0]_{5 \times 1}} \\
-[1]_{5 \times 1}
\end{array}\right],
$$

where $[0]_{n \times m}$ represents a zero-matrix of the indicated dimensions, $I_{n}$ is the identity matrix of order $n$, and $[1]_{n \times 1}$ is a vector of dimension $n$ with all its entries equal to 1 . Next, we consider the vector of interstory drifts

$$
x_{r}(t)=\left[q_{1}, q_{2}-q_{1}, q_{3}-q_{2}, q_{4}-q_{3}, q_{5}-q_{4}\right]^{T},
$$

and the vector of interstory velocities $v_{r}(t)$ given in (9) to define the new state vector

$$
x(t)=\left[\begin{array}{l}
x_{r}(t) \\
v_{r}(t)
\end{array}\right],
$$

which can be expressed as $x(t)=\mathcal{C} x_{I}(t)$ with

$$
\mathcal{C}=\left[\begin{array}{rrrrrrrrrr}
1 & 0 & 0 & 0 & 0 & 0 & 0 & 0 & 0 & 0 \\
-1 & 1 & 0 & 0 & 0 & 0 & 0 & 0 & 0 & 0 \\
0 & -1 & 1 & 0 & 0 & 0 & 0 & 0 & 0 & 0 \\
0 & 0 & -1 & 1 & 0 & 0 & 0 & 0 & 0 & 0 \\
0 & 0 & 0 & -1 & 1 & 0 & 0 & 0 & 0 & 0 \\
0 & 0 & 0 & 0 & 0 & 1 & 0 & 0 & 0 & 0 \\
0 & 0 & 0 & 0 & 0 & -1 & 1 & 0 & 0 & 0 \\
0 & 0 & 0 & 0 & 0 & 0 & -1 & 1 & 0 & 0 \\
0 & 0 & 0 & 0 & 0 & 0 & 0 & -1 & 1 & 0 \\
0 & 0 & 0 & 0 & 0 & 0 & 0 & 0 & -1 & 1
\end{array}\right]
$$

The new state-space model is

$$
S: \dot{x}(t)=A x(t)+B u(t)+E w(t),
$$

with $A=\mathcal{C} A_{I} \mathcal{C}^{-1}, B=\mathcal{C} B_{I}$, and $E=\mathcal{C} E_{I}$. The particular values of the matrices $A, B$ and $E$ are presented in Figure 4.

\section{B. State-feedback $H_{\infty}$ controller design}

In this subsection, we assume that the actuation devices $a_{i}$ displayed in Figure 2 are ideal force actuators and we design a state-feedback $H_{\infty}$ controller

$$
u(t)=G_{s} x(t)
$$

to drive the actuation system. By setting the output matrix $C_{y}=I_{10}$ in (17), the control design methodology discussed in Section III can be applied to compute the control gain matrix $G_{s}$. In this case, solving the convex optimization problem (28) leads to the equation

$$
\tilde{Y}=G_{s} \tilde{X}
$$




$$
\begin{aligned}
& A=10^{3} \times\left[\begin{array}{cccccccccc}
0 & 0 & 0 & 0 & 0 & 0.0010 & 0 & 0 & 0 & 0 \\
0 & 0 & 0 & 0 & 0 & 0 & 0.0010 & 0 & 0 & 0 \\
0 & 0 & 0 & 0 & 0 & 0 & 0 & 0.0010 & 0 & 0 \\
0 & 0 & 0 & 0 & 0 & 0 & 0 & 0 & 0.0010 & 0 \\
0 & 0 & 0 & 0 & 0 & 0 & 0 & 0 & 0 & 0.0010 \\
-0.6831 & 0.5251 & 0 & 0 & 0 & -0.0008 & 0.0004 & 0 & 0 & 0 \\
0.6831 & -1.0652 & 0.4732 & 0 & 0 & 0.0006 & -0.0011 & 0.0004 & 0 & 0 \\
0 & 0.5402 & -0.9515 & 0.4300 & 0 & 0 & 0.0004 & -0.0010 & 0.0004 & 0 \\
0 & 0 & 0.4783 & -0.8645 & 0.4102 & 0 & 0 & 0.0004 & -0.0009 & 0.0003 \\
0 & 0 & 0 & 0.4346 & -0.7258 & 0 & 0 & 0 & 0.0004 & -0.0008
\end{array}\right] \\
& B=10^{-5} \times\left[\begin{array}{ccccc}
0 & 0 & 0 & 0 & 0 \\
0 & 0 & 0 & 0 & 0 \\
0 & 0 & 0 & 0 & 0 \\
0 & 0 & 0 & 0 & 0 \\
0 & 0 & 0 & 0 & 0 \\
0.4647 & -0.4647 & 0 & 0 & 0 \\
-0.4647 & 0.9427 & -0.4780 & 0 & 0 \\
0 & -0.4780 & 0.9611 & -0.4831 & 0 \\
0 & 0 & -0.4831 & 0.9714 & -0.4883 \\
0 & 0 & 0 & -0.4883 & 0.8641
\end{array}\right], \quad E=\left[\begin{array}{c}
0 \\
0 \\
0 \\
0 \\
0 \\
-1 \\
0 \\
0 \\
0 \\
0
\end{array}\right]
\end{aligned}
$$

Fig. 4. System matrices of the first-order state-space model with interstory drifts and interstory velocities as state variables

$$
G_{s}=10^{6} \times\left[\begin{array}{rrrrrrrrrr}
3.7829 & -0.2483 & 1.4222 & -1.5182 & -0.9603 & -1.6909 & -0.5176 & -0.7646 & -0.4627 & -0.2493 \\
-4.1728 & 6.4541 & -1.4370 & 1.6774 & -0.7418 & -1.3895 & -1.0085 & -0.8411 & -0.6914 & -0.3860 \\
1.9275 & -5.3112 & 6.3232 & 2.7760 & -0.3177 & -0.9987 & -0.9974 & -1.1183 & -0.7537 & -0.2999 \\
2.8607 & -0.7861 & -5.2123 & 5.8457 & -2.2138 & -0.7892 & -0.7467 & -0.7907 & -0.9274 & -0.3838 \\
1.6547 & 0.2569 & -1.0827 & -4.7663 & 6.4189 & -0.5497 & -0.5086 & -0.5161 & -0.4060 & -0.3641
\end{array}\right]
$$

Fig. 5. State-feedback control gain matrix $G_{s}$

which can be easily solved for $G_{s}$, resulting

$$
G_{s}=\tilde{Y}(\tilde{X})^{-1}
$$

For the controlled-output matrices

$$
C_{z}=\left[\begin{array}{c}
I_{10} \\
{[0]_{5 \times 10}}
\end{array}\right], \quad D_{z}=10^{-6.2} \times\left[\begin{array}{c}
{[0]_{10 \times 5}} \\
I_{5}
\end{array}\right],
$$

the optimization problem (28) produces the control gain matrix $G_{s}$ displayed in Figure 5 with $H_{\infty}$-norm

$$
\gamma_{G_{s}}=0.9124
$$

\section{Design of the passive damping system}

Now, let us suppose that the actuation devices $a_{i}$ in Figure 2 are linear passive dampers with adjustable damping constants $\hat{c}_{i}$. According to the discussion in Section II, this passive damping system can be suitably tuned by designing a decentralized velocity-feedback controller $u(t)=G_{d} y(t)$ with $y(t)=\dot{v}_{r}(t)$, and taking the damping constants $\hat{c}_{i}=$ $-\left[g_{d}\right]_{i i}, 1 \leq i \leq 5$, where $\left[g_{d}\right]_{i i}$ are the elements of the diagonal matrix $G_{d}$.

To this end, we consider the model (17) with the output matrix $C_{y}=\left[[0]_{5 \times 5} I_{5}\right]$, together with the matrices $C_{z}$ and $D_{z}$ given in (52). Next, we solve the optimization problem (34) constraining the LMI matrices $X_{R}$ and $Y_{R}$ to diagonal form in order to compute a diagonal gain matrix $G_{d}$. It should be noted that a first attempt at solving the LMI optimization problem with the Matlab Robust Control Toolbox [12] fails, and the problem is reported to be infeasible. However, as pointed out in [7], this difficulty can be conveniently circumvented by adding a small perturbation to the system matrix. Using the perturbed state matrix $\hat{A}=$ $A+\Delta A$ with $\Delta A=-0.01 \times I_{10}$, the following decentralized velocity-feedback control matrix results:

$$
G_{d}=10^{6} \times\left[\begin{array}{ccccc}
-5.1663 & 0 & 0 & 0 & 0 \\
0 & -3.345 & 0 & 0 & 0 \\
0 & 0 & -2.725 & 0 & 0 \\
0 & 0 & 0 & -2.294 & 0 \\
0 & 0 & 0 & 0 & -2.084
\end{array}\right]
$$

which defines a passive damping system with damping constants

$$
\begin{aligned}
& \hat{c}_{1}=5.1663 \times 10^{6}, \hat{c}_{2}=3.345 \times 10^{6}, \hat{c}_{3}=2.725 \times 10^{6}, \\
& \hat{c}_{4}=2.294 \times 10^{6}, \hat{c}_{5}=2.084 \times 10^{6} .
\end{aligned}
$$

The optimal $\gamma$-value obtained in the solution of the LMI optimization problem is $\gamma_{d}=\left(\tilde{\eta}_{\text {opt }}\right)^{-1 / 2}=0.9522$.

\section{NUMERICAL SIMULATIONS}

In this section, the full-scale North-South 1940 El Centro seismic record (see Figure 6) is used as ground acceleration input to carry out numerical simulations of the free and controlled responses of the five-story building. In Figure 7, the upper graphic shows the maximum absolute interstory drifts obtained for three different configurations: (i) uncontrolled building (black squares), (ii) controlled building with ideal force actuation devices driven by the centralized state-feedback controller defined by the gain matrix $G_{s}$ (blue circles), and (iii) controlled building with the passive damping system defined by the damping constants given in (55) (red asterisks). These configurations are denoted in the legend as Free, State-feedback and Passive, respectively. 


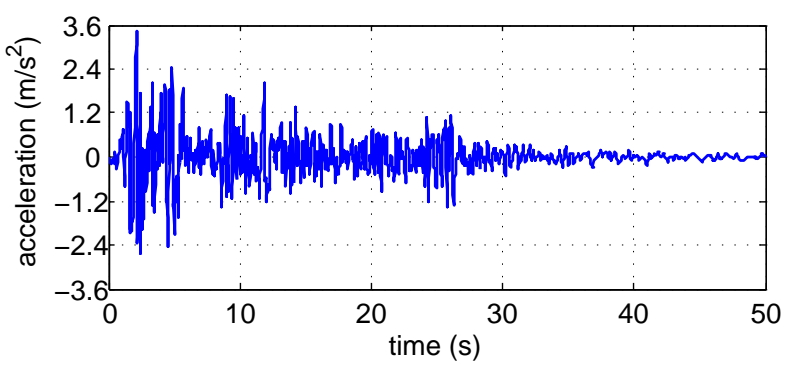

Fig. 6. Full-scale North-South 1940 El Centro seismic record
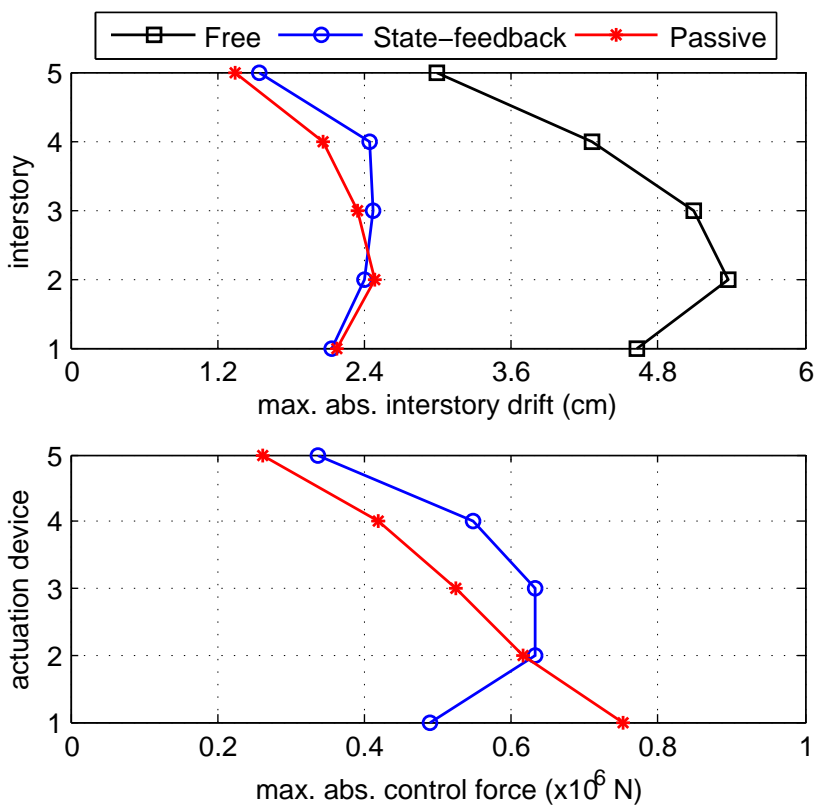

Fig. 7. Maximum absolute interstory drifts and control efforts

The corresponding maximum absolute actuation forces are displayed in the lower graphic using the same symbols and colors. Together with the typical good behavior of the state-feedback $H_{\infty}$ controllers, the graphics show that the passive damping system achieves practically the same maximum absolute interstory drifts as the state-feedback controller, requiring also similar levels of control effort. The behavior exhibited by the passive damping system is certainly remarkable, especially if we take into account that it can operate without sensors, with null power requirements, and no communication system.

\section{FinAl REMARKS AND FUtURE DiRECTIONS}

The particular example of passive-damping design presented in this paper has been carried out following an $H_{\infty}$ approach. However, it has to be noted that the proposed methodology can also produce positive results for other control strategies that can be formulated in terms of optimization problems with LMI constraints. It should also be highlighted that the new approach can be of interest in a wide variety of research fields where the mitigation of undesirable vibrational responses is a major concern. Examples of practical interest can be found, for instance, in seismic protection of multibuilding systems [13]-[15], automotive industry [16]-[18], or offshore wind power generation [19], [20]. Consequently, further research effort needs to be aimed at exploring additional applications of the proposed methodology.

\section{REFERENCES}

[1] B. Spencer and S. Nagarajaiah, "State of the art of structural control," Journal of Structural Engineering, vol. 129, no. 7, pp. 845-856, July 2003.

[2] Y. Ikeda, "Active and semi-active vibration control of buildings in Japan - Practical applications and verification," Structural Control and Health Monotoring, vol. 16, no. 7-8, pp. 703-723, 2009.

[3] H. Li and L. Huo, "Advances in structural control in civil engineering in China," Mathematical Problems in Engineering, vol. 2010, pp. 123, 2010.

[4] N. Gluck, A. Reinhorn, J. Gluck, and R. Levy, "Design of supplemental dampers for control of structures," Journal of Structural Engineering, vol. 122, no. 12, pp. 1394-1399, 1996.

[5] A. Agrawal and J. Yang, "Design of passive energy dissipation systems based on LQR control methods," Journal of Intelligent Material Systems and Structures, vol. 10, no. 12, pp. 933-944, 1999.

[6] J. Yang, S. Lin, J.-H. Kim, and A. Agrawal, "Optimal design of passive energy dissipation systems based on $H_{\infty}$ and $H_{2}$ performances," Earthquake Engineering and Structural Dynamics, vol. 31, no. 4, pp. 921-936, 2002.

[7] J. Rubió-Massegú, F. Palacios-Quiñonero, and J. Rossell, "Decentralized static output-feedback $H_{\infty}$ controller design for buildings under seismic excitation," Earthquake Engineering and Structural Dynamics, vol. 41, pp. 1199-1205, 2012.

[8] J. Rubió-Massegú, J. Rossell, H. Karimi, and F. Palacios-Quiñonero, "Static output-feedback control under information structure constraints," Automatica, vol. 49, no. 1, pp. 313-316, 2013.

[9] S. Boyd, L. E. Ghaoui, E. Feron, and V. Balakrishnan, Linear Matrix Inequalities in System and Control Theory. Philadelphia, USA: SIAM Studies in Applied Mathematics, 1994.

[10] N. Kurata, T. Kobori, M. Takahashi, N. Niwa, and H. Midorikawa, "Actual seismic response controlled building with semi-active damper system," Earthquake Engineering and Structural Dynamics, vol. 28, no. 11, pp. 1427-1447, 1999.

[11] A. Chopra, Dynamics of Structures. Theory and Applications to Earthquake Engineering, 3rd ed. Upper Saddle River, New Jersey, USA: Prentice Hall, 2007.

[12] G. Balas, R. Chiang, A. Packard, and M. Safonov, MATLAB ${ }^{T M}$ Robust Control Toolbox ${ }^{T M}$ 3. User's Guide, The MathsWorks, Inc., 3 Apple Hill Drive. Natick, MA 01760-20, USA, 2011.

[13] H. Zhu, D. Ge, and X. Huang, "Optimum connecting dampers to reduce the seismic responses of parallel structures," Journal of Sound and Vibration, vol. 330, no. 9, pp. 1931-1949, 2011.

[14] F. Palacios-Quiñonero, J. Rubió-Massegú, J. Rossell, and H. Karimi, "Semiactive-passive structural vibration control strategy for adjacent structures under seismic excitation," Journal of the Franklin Institute, vol. 349, no. 10, pp. 3003-3026, 2012.

[15] F. Palacios-Quiñonero, J. Rossell, J. Rubió-Massegú, and H. Karimi, "Structural vibration control for a class of connected multistructure mechanical systems," Mathematical Problems in Engineering, vol. 2012, pp. 1-23, 2012.

[16] H. Zhang, Y. Shi, and A. Mehr, "Robust non-fragile dynamic vibration absorbers with uncertain factors," Journal of Sound and Vibration, vol. 330, no. 4, pp. 559-566, 2011.

[17] H. Li, H. Liu, H. Gao, and P. Shi, "Reliable fuzzy control for active suspension systems with actuator delay and fault," IEEE Transactions on Fuzzy Systems, vol. 20, no. 2, pp. 342-357, 2012.

[18] M. Zapateiro, F. Pozo, H. Karimi, and N. Luo, "Semiactive control methodologies for suspension control with magnetorheological dampers," IEEE/ASME Transactions on Mechatronics, vol. 17, no. 2, pp. 370-380, 2012.

[19] S. Colwell and B. Basu, "Tuned liquid column dampers in offshore wind turbines for structural control," Engineering Structures, vol. 31, no. 2, pp. 358-368, 2009.

[20] J. Li, Z. Zhang, and J. Chen, "Experimental study on vibration control of offshore wind turbines usign a ball vibration absorber," Energy and Power Engineering, vol. 4, pp. 153-157, 2012. 